\title{
Bispectral Analysis of Spontaneous EEG Activity from Patients with Moderate Dementia Due to Alzheimer's Disease
}

\author{
Carlos Gómez, IEEE Senior Member, Fernando Vaquerizo-Villar, Jesús Poza, IEEE Senior Member, \\ Saúl J. Ruiz, Miguel A. Tola-Arribas, Mónica Cano, and Roberto Hornero, IEEE Senior Member
}

\begin{abstract}
Dementia due to Alzheimer's disease (AD) is a common disorder with a great impact on the patients' quality of life. The aim of this pilot study was to characterize spontaneous electroencephalography (EEG) activity in dementia due to AD using bispectral analysis. Five minutes of EEG activity were recorded from 17 patients with moderate dementia due to AD and 19 age-matched controls. Bispectrum results revealed that $A D$ patients are characterized by an increase of phase coupling at low frequencies in comparison with controls. Additionally, some bispectral features calculated from the bispectrum showed significant differences between both groups $(p<0.05$, Mann-Whitney $U$ test with Bonferroni's correction). Finally, a stepwise logistic regression analysis with a leave-one-out cross-validation procedure was used for classification purposes. An accuracy of $86.11 \%$ (sensitivity = $88.24 \%$; specificity $=84.21 \%$ ) was achieved. This study suggests the usefulness of bispectral analysis to provide further insights into the underlying brain dynamics associated with AD.
\end{abstract}

\section{INTRODUCTION}

Dementia is the main cause of long-term functional dependence in elderly population [1]. The global prevalence of dementia was 24 million in 2011, and it is estimated to double every 20 years through to 2040 [2]. Dementia due to Alzheimer's disease (AD) is a common disorder with a huge impact on the quality of life of the patients and their caregivers [3]. For all these reasons, dementia due to $\mathrm{AD}$ is becoming a key public-healthcare and economic problem. Pathologically, AD is characterized by the presence of extracellular amyloid beta-peptide plaques and intracellular tau aggregation [4]. Currently, a definite diagnosis of AD could only be made upon death, after examination of tissue samples at autopsy. For this reason, physicians can really only make a diagnosis of probable AD. Clinical diagnosis usually includes a thorough medical history, mental status tests, physical and neurological examinations, cerebrospinal fluid biomarkers, blood tests, and neuroimaging studies. Nowadays, the National Institute on Aging and Alzheimer's Association (NIA-AA) does not recommend

This research was supported in part by 'Ministerio de Economía y Competitividad (MINECO)' and FEDER under project TEC2014-53196-R, and 'Consejería de Educación de la Junta de Castilla y León' under project VA037U16. F. Vaquerizo-Villar was in receipt of a 'Promoción de Empleo Joven e Implantación de la Garantía Juvenil en $\mathrm{I}+\mathrm{D}+\mathrm{i}$ ' grant from MINECO and University of Valladolid.

C. Gómez, F. Vaquerizo-Villar, J. Poza, Saúl J. Ruiz, and R. Hornero are with the Biomedical Engineering Group, E.T.S. Ingenieros de Telecomunicación, University of Valladolid, Paseo Belén 15, 47011 Valladolid, Spain (e-mail: carlos.gomez@tel.uva.es).

M. A. Tola is with the Department of Neurology, Hospital Universitario Río Hortega, Valladolid, Spain.

M. Cano is with the Department of Clinical Neurophysiology, Hospital Universitario Río Hortega, Valladolid, Spain. electroencephalography (EEG) for the diagnosis of dementia due to $\mathrm{AD}$, in spite of its potential to characterize neural dynamics [5]. EEG is a non-invasive technique to measure the electromagnetic brain activity [6]. It allows the assessment of the spatio-temporal patterns of neural activity and their interactions in the time range of milliseconds [7]. As AD affects different cerebral cortex areas, EEG is indeed a useful technique to understand the neural mechanisms of this disorder.

In the last decades, much effort has been devoted to characterize the neural activity in AD. Spectral techniques are the most extended option to analyze EEG activity from AD patients, due to their simplicity and ease of clinical interpretation. The most common marker in AD patients' EEG is an increased brain activity at lower frequency bands [8]. Spectral entropies and spectral ratios have been also applied in order to characterize the neural activity in AD $[9,10]$. However, the phase relationship among components is lost when the EEG is processed with these classical spectral measures [11]. Additionally, these measures assume stationarity, whereas EEG is essentially a non-stationary signal. Therefore, new methods are needed to overcome the limitations of the aforementioned analyses.

Bispectral analysis is a suitable tool to track the changes in signals arising from both nonlinear and linear changes in the generating process [12]. The motivations to use this signal processing technique are the following [11,13]: (i) to measure non-Gaussianity (normality); (ii) to obtain information about the phase of the time series; and (iii) to detect and characterize the nonlinear mechanisms that generate time series via phase relations of their harmonic components. In spite of its potential, only a few studies applied measures related to the bispectrum to characterize the brain dynamics in AD. For instance, Samar et al. [14] suggested that quadratic phase coupling patterns in delta band were altered in this form of dementia. Other study concluded that a significant proportion of patients with dementia shows a low baseline value of the Bispectral Index [15]. The aim of this study is to characterize EEG changes associated with the dementia due to $\mathrm{AD}$, using five features extracted from the bispectrum: two bispectral entropies and three features related to the third-order statistics.

\section{MATERIALS}

\section{A. Subjects}

EEG signals were recorded from 36 subjects. The AD group was formed by 17 patients with moderate dementia due to $\mathrm{AD}$, diagnosed according to the clinical criteria of the NIA-AA. Nineteen elderly subjects without cognitive impairments and with no history of neurological or 
TABLE I. SOCIO-DEMOGRAPHIC AND CLINICAL DATA FOR EACH GROUP

\begin{tabular}{|c|c|c|}
\cline { 2 - 3 } \multicolumn{1}{c|}{} & AD group & Control group \\
\hline Number of subjects & 17 & 19 \\
\hline Age (years) (Mean \pm SD) & $79.6 \pm 5.6$ & $77.0 \pm 3.9$ \\
\hline Gender (Male:Female) $^{\circ}$ & $5: 12$ & $9: 10$ \\
\hline MMSE $^{\text {a }}$ (Mean \pm SD) & $16.0 \pm 2.5$ & $28.6 \pm 1.3$ \\
\hline B-ADL $^{\text {b }}$ (Mean \pm SD) & $6.3 \pm 1.7$ & $1.4 \pm 0.8$ \\
\hline & \multicolumn{3}{|c|}{${ }^{\mathrm{b}}$ MMSE: Mini-Mental State Examination } \\
\hline
\end{tabular}

psychiatric disorders comprised the control group. Table 1 shows socio-demographic and clinical data for both groups. It is important to note that no significant difference in age was found between controls and patients. All participants and patients' caregivers gave their written informed consent to be included in the study. The Ethics Committee at the "Hospital Universitario Río Hortega" (Valladolid, Spain) endorsed the study protocol, according to The Code of Ethics of the World Medical Association (Declaration of Helsinki).

\section{B. EEG recording}

EEG activity was recorded with a 19-channel EEG equipment (XLTEK ${ }^{\circledR}$, Natus Medical). This system uses the International 10-20 System with 19-channels standard electrode layout: Fp1, Fp2, Fz, F3, F4, F7, F8, Cz, C3, C4, T3, T4, T5, T6, Pz, P3, P4, O1 and O2. For each subject, five minutes of spontaneous EEG activity were acquired at a sampling frequency of $200 \mathrm{~Hz}$. Afterwards, EEG data were digitally filtered between 1 and $40 \mathrm{~Hz}$. Finally, artifact-free epochs of 5-s length were selected by visual inspection by an experienced EEG investigator.

\section{METHODS}

\section{A. Bispectrum}

Higher order spectra (HOS), also called higher order statistics, are defined as the Fourier transformations of higher

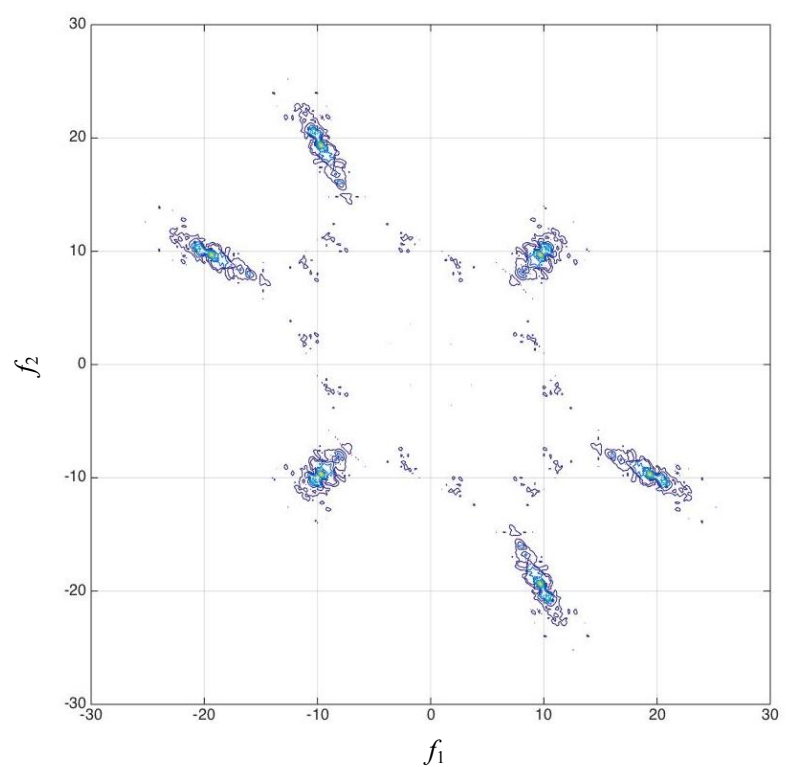

order cumulants of a random process [11]. A particular example of HOS is power spectrum, which is defined to be the Fourier transform of the second-order cumulant sequence. On the other hand, bispectrum and trispectrum correspond with the third- and the fourth-order cumulant spectra. In this study, EEG signals were analyzed using bispectrum. The bispectrum can be considered as a decomposition of the third moment (skewness) of a signal over frequency. The usefulness of bispectrum to analyze systems with asymmetric non-linearities has been already demonstrated [16].

Let $x(n)$ be a deterministic and zero-mean time series, its bispectrum may be expressed in terms of the Fourier transform of the signal as [11]:

$$
\begin{aligned}
B\left(f_{1}, f_{2}\right)= & \sum_{\tau_{1}=-\infty}^{\infty} \sum_{\tau_{2}=-\infty}^{\infty} \sum_{n=-\infty}^{\infty} x(n) \cdot x\left(n+\tau_{1}\right) \cdot x\left(n+\tau_{2}\right) \cdot \\
& \cdot \exp \left[i 2 \pi \cdot\left(f_{1} \tau_{1}+f_{2} \tau_{2}\right)\right] .
\end{aligned}
$$

Setting $n+\tau_{1}=m$ and $n+\tau_{2}=k$, and splitting the exponent, equation 1 can be expressed as:

$$
\begin{aligned}
B\left(f_{1}, f_{2}\right)= & \left\{\sum_{m=-\infty}^{\infty} x(m) e^{-i 2 \pi f_{1} m}\right\} \cdot\left\{\sum_{k=-\infty}^{\infty} x(k) e^{-i 2 \pi f_{2} k}\right\} . \\
& \cdot\left\{\sum_{n=-\infty}^{\infty} x(n) e^{+i 2 \pi\left(f_{1}+f_{2}\right) n}\right\}= \\
= & X\left(\omega_{1}\right) \cdot X\left(\omega_{2}\right) \cdot X^{*}\left(\omega_{1}+\omega_{2}\right) .
\end{aligned}
$$

Equation 2 is a triple product evaluated at two frequencies, $f_{1}$ and $f_{2}$, and their sum, $f_{1}+f_{2}$. This expression is analogous to the periodogram for power spectrum. For this reason, it is called higher order periodogram [11]. Figure 1 shows the contour plots of the grand-averaged higher order periodograms for control and $\mathrm{AD}$ groups. It is important to note that just as the power spectrum has symmetrical properties, so do the bispectrum [16]. Therefore, it is only

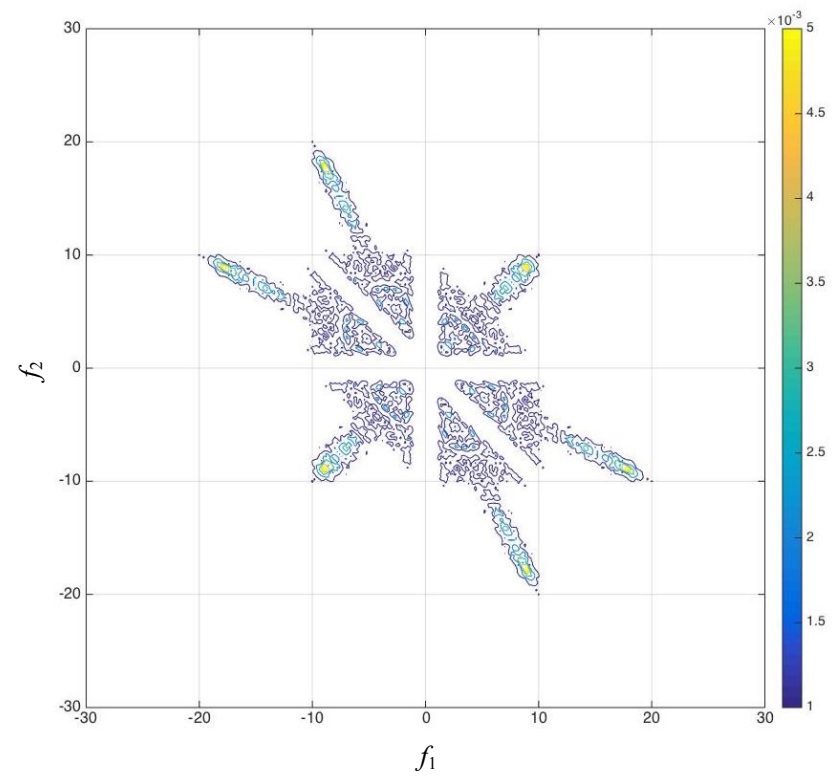

Figure 1. Contour plots of the grand-averaged higher order periodograms for control subjects (left panel) and AD patients (right panel). 
necessary to evaluate the bispectrum in the triangular region that satisfies $f_{2} \geq 0, f_{2} \geq f_{1}$ and $f_{1}+f_{2} \leq f_{s} / 2$ [11]. This region is known as the principal domain of the bispectrum [16].

\section{B. Bispectral features}

After the computation of bispectrum, different bispectral features can be extracted. Several bispectral features have been already proposed in the literature: mean magnitude of the bispectrum, phase entropy, weighted center of the bispectrum, and relative power of the bispectrum, among others $[11,17]$. In this study, five features were calculated: normalized bispectral entropy $\left(P_{1}\right)$, normalized bispectral squared entropy $\left(P_{2}\right)$, and three features related to the thirdorder statistics $\left(H_{1}, H_{2}\right.$ and $\left.H_{3}\right)$. Let be $B\left(f_{1}, f_{2}\right)$ the bispectrum in the principal domain of an EEG epoch $x(n)$ and given a frequency range $F$ (in this study, $F$ corresponds to the typical five EEG frequency bands: delta, theta, alpha, beta, and gamma), the following bispectral features were computed $[18,19]$ :

- $\quad$ Normalized bispectral entropy $\left(P_{1}\right)$ :

$$
P_{1}=-\sum_{i} p_{i} \log p_{i},
$$

where

$$
p_{i}=\frac{\left|B\left(f_{1}, f_{2}\right)\right|}{\sum_{f_{1}, f_{2} \in F} \in\left(f_{1}, f_{2}\right) \mid}, \quad f_{1}, f_{2} \in F .
$$

Each $p_{i}$ corresponds to each combination of $f_{1}$ and $f_{2}$ values.

- $\quad$ Normalized bispectral squared entropy $\left(P_{2}\right)$ :

$$
P_{2}=-\sum_{j} p_{j} \log p_{j}
$$

where

$$
p_{j}=\frac{\left|B\left(f_{1}, f_{2}\right)\right|^{2}}{\sum_{f_{1}, f_{2} \in F}\left|B\left(f_{1}, f_{2}\right)\right|^{2}}, \quad f_{1}, f_{2} \in F .
$$

Each $p_{j}$ corresponds to each combination of $f_{1}$ and $f_{2}$ values.

- Sum of logarithmic amplitudes of the bispectrum $\left(H_{1}\right)$ :

$$
H_{1}=\sum_{f_{1}, f_{2} \in F} \log \left(\left|B\left(f_{1}, f_{2}\right)\right|\right),
$$

- Sum of logarithmic amplitudes of diagonal elements of the bispectrum $\left(\mathrm{H}_{2}\right)$ :

$$
H_{2}=\sum_{k=1}^{N} \log \left(\left|B\left(f_{k}, f_{k}\right)\right|\right), \quad f_{k} \in F .
$$

- First-order spectral moment of the amplitudes of diagonal elements of the bispectrum $\left(\mathrm{H}_{3}\right)$ :

$$
H_{3}=\sum_{k=1}^{N} k \cdot \log \left(\left|B\left(f_{k}, f_{k}\right)\right|\right), \quad f_{k} \in F .
$$

The above 5 features were estimatated for each artifactfree epoch, EEG channel, and frequency band. Then, results were averaged over epochs and channels, obtained only one value of $P_{1}, P_{2}, H_{1}, H_{2}$, and $H_{3}$ per subject and frequency band.

\section{Statistical analysis}

Kolmogorov-Smirnov and Shapiro-Wilk tests were used to assess normality of distribution, whereas Levene's test was applied to verify the homocedasticity. As data did not meet parametric test assumptions, statistical comparisons between groups were performed by means of Mann-Whitney $U$ test.

Additionally, receiver operating characteristic (ROC) curves were used to evaluate the ability of each bispectral feature to distinguish between both groups. In order to calculate sensitivity, specificity, accuracy, and area under the curve (AUC) values, a leave-one-out cross-validation (LOOCV) procedure was used. Lastly, a stepwise logistic regression analysis, also with a LOOCV procedure, was used to build the multivariate model for the classification of the subjects into $\mathrm{AD}$ or control groups.

\section{RESULTS AND DISCUSSION}

\section{A. Bispectrum}

In this study, bispectrum was applied to the EEG data from 17 patients with dementia due to $\mathrm{AD}$ and 19 elderly control subjects. This technique was proposed in order to recover information regarding deviation from Gaussianity, but also to detect the presence of phase couplings [20]. This is an important advantage of bispectrum over classical spectral measures [20]. In the current study, AD patients showed an increase of phase coupling at low frequencies in comparison with controls (see Fig. 1). These findings agree with previous studies [21, 22]. For instance, phase lag index, a novel measure to quantify phase sychronization, was applied to magnetoencephalography recordings from AD patients and controls. It revealed higher phase synchronization in AD group than in control one at delta and theta bands. The opposite behavior was found in high frequency bands [22]. In other study, Koenig et al. [21] applied global field synchronization, which reflects the global amount of phase-locked activity at a given frequency, to a large EEG database. Their results showed that AD patients are associated with decreased coupling values in alpha, beta and gamma frequency bands, and increased values in delta. All these studies support the hypothesis that AD includes a disconnection syndrome [23].

\section{B. Bispectral features}

Five bispectral features $\left(P_{1}, P_{2}, H_{1}, H_{2}\right.$, and $\left.H_{3}\right)$ were calculated for each epoch. After the averaging process over all artifact-free epochs and channels, only one value of each feature per subject and frequency band was obtained. Statistical analyses revealed significant differences $(p<0.05$, Mann Whitney $U$ test) between controls and $\mathrm{AD}$ patients for $H_{1}, H_{2}$, and $H_{3}$ features at delta, theta, and alpha frequency bands, and only for $\mathrm{H}_{2}$ in beta band (see Table 2). However, only features $H_{1}$ and $H_{2}$ in theta, and $H_{3}$ in alpha remained significant after a Bonferroni's correction.

ROC curves with a LOOCV procedure were used to assess the ability of bispectral features to discriminate patients with dementia due to $\mathrm{AD}$ from control subjects. The highest accuracy value was obtained for $\mathrm{H}_{2}$ feature at theta frequency band: $83.33 \%$ (sensitivity $=83.25 \%$; specificity $=$ $84.21 \%$; AUC $=0.84$ ). Finally, a stepwise logistic regression model was created based on the following variables: $\mathrm{H}_{2}$ (theta), $H_{1}$ (alpha), $H_{2}$ (alpha), and $H_{3}$ (alpha). With this model, an accuracy of $86.11 \%$ was achieved (sensitivity = $88.24 \%$; specificity $=84.21 \%$; AUC $=0.93$ ). This value compares well with the accuracies reported by previous studies $[9,24]$. 


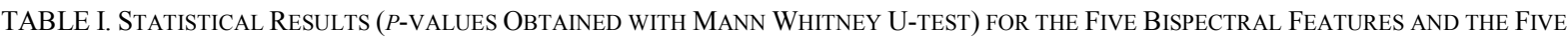
FREQUENCY BANDS. SIGNIFICANT DIFFERENCES ARE DISPLAYED IN ITALICS.

\begin{tabular}{|c|c|c|c|c|c|}
\cline { 2 - 5 } \multicolumn{1}{c|}{} & $\boldsymbol{P}_{\mathbf{1}}$ & $\boldsymbol{P}_{\mathbf{2}}$ & $\boldsymbol{H}_{\mathbf{1}}$ & $\boldsymbol{H}_{\mathbf{2}}$ & $\boldsymbol{H}_{\mathbf{3}}$ \\
\hline Delta & 0,3106 & 0,3106 & 0,0190 & 0,0207 & 0,0058 \\
\hline Theta & 0,0532 & 0,0760 & 0,0009 & 0,0005 & 0,0026 \\
\hline Alpha & 0,1939 & 0,2165 & 0,0071 & 0,0048 & 0,0015 \\
\hline Beta & 0,4661 & 0,9495 & 0,0532 & 0,0459 & 0,0814 \\
\hline Gamma & 0,4283 & 0,5901 & 0,4100 & 0,4100 & 0,3581 \\
\hline
\end{tabular}

\section{Limitations and future work}

Some limitations of our research work should be noted. Firstly, only 17 patients with moderate dementia due to $\mathrm{AD}$ and 19 elderly controls took part in this study. Future efforts will be focused to increase the number of subjects, as well as to include new groups: subjects with mild cognitive impairment and patients with mild dementia due to $\mathrm{AD}$. Secondly, only interactions within the typical EEG frequency bands have been analyzed. However, interaction between different frequency bands can provide complementary information. Finally, only five bispectral features have been calculated in this study, but other features could also help to understand the mechanisms underlying this complex disorder.

\section{CONCLUSION}

Our study leads us to conclude that spontaneous EEG activity in patients with dementia due to $\mathrm{AD}$ is characterized by changes in the higher order periodogram. This study suggests the usefulness of our approach (five different bispectral features calculated at different frequency bands) to provide further insights into the underlying brain dynamics associated with $\mathrm{AD}$.

\section{REFERENCES}

[1] H. Agüero-Torres, L. Fratiglioni, Z. Guo, M. Viitanen, E. von Strauss, and B. Winblad, "Dementia is the major cause of functional dependence in the elderly: 3-year follow-up data from a populationbased study," Am. J. Public Health, vol. 88, pp. 1452-1456, 1998.

[2] C. Reitz, C. Brayne, and R. Mayeux, "Epidemiology of Alzheimer disease," Nat. Rev. Neurol., vol. 7, pp. 137-152, 2011.

[3] A. Paganini-Hill and V. W. Henderson, "Estrogen deficiency and risk of Alzheimer's disease in women," Am. J. Epidemiol., vol. 140, pp. 256-261, 1994.

[4] B. P. Lucey and D. M. Holtzman, "How amyloid, sleep and memory connect," Nature Neurosci., vol. 18, pp. 933-934, 2015.

[5] J. Jeong, "EEG dynamics in patients with Alzheimer's disease," Clin. Neurophysiol., vol. 115, pp. 1490-1505, 2004.

[6] B. J. Roach and D. H. Mathalon, "Event-related EEG time-frequency analysis: an overview of measures and an analysis of early gamma band phase locking in schizophrenia," Schizophr. Bull., vol. 34, pp. 907-926, 2008.

[7] T. P. Meehan and S. L. Bressler, "Neurocognitive networks: Findings, models, and theory," Neurosci. Biobehav. R., vol. 36, pp. 2232-2247, 2012.

[8] M. Signorino, E. Pucci, N. Belardinelli, G. Nolfe, and F. Angeleri, "EEG spectral analysis in vascular and Alzheimer dementia," Electroencephalogr. Clin. Neurophysiol., vol. 94, pp. 313-325, 1995.

[9] D. Abásolo, R. Hornero, P. Espino, D. Alvarez, and J. Poza, "Entropy analysis of the EEG background activity in Alzheimer's disease patients," Physiol. Meas., vol. 27, pp. 241-253, 2006.
[10] J. Poza, R. Hornero, D. Abásolo, A. Fernández, and A. Mayo, "Evaluation of spectral ratio measures from spontaneous MEG recordings in patients with Alzheimer's disease," Comput. Methods Programs Biomed., vol. 90, pp. 137-147, 2008.

[11] K. C. Chua, V. Chandran, U. R. Acharya, and C. M. Lim, "Application of higher order statistics/spectra in biomedical signals-A review," Med. Eng. Phys. vol. 32, pp. 679-689, 2010.

[12] J. C. Sigl and N. G. Chamoun, "An introduction to bispectral analysis for the electroencephalogram," J. Clin. Monit. Comput., vol. 10, pp. 392-404, 1994.

[13] J. W. Zhang, C. X. Zheng, and A. Xie, "Bispectrum analysis of focal ischemic cerebral EEG signal using third-order recursion method," IEEE Trans. Biomed. Eng., vol. 47, pp. 352-359, 2000.

[14] V. J. Samar, K. P. Swartz, M. R. Raghuveer, A. L. Braveman, J. M. Centola, and J. K. Jackson, "Quadratic phase coupling in auditory evoked potentials from healthy old subjects and subjects with Alzheimer's dementia," in: IEEE Signal Processing Workshop on Higher-Order Statistics, pp. 361-365, 1993.

[15] M. Renna, J. Handy, and A. Shah, "Low baseline bispectral index of the electroencephalogram in patients with dementia," Anesth. Analg., vol. 96, pp. 1380-1385, 2003.

[16] W. B. Collis, P. R. White, and J. K. Hammond, "Higher-order spectra: the bispectrum and trispectrum," Mech. Syst. Signal Process., vol. 12, pp. 375-394, 1998.

[17] G. Valenza, A. Lanata, and E. P. Scilingo, "The role of nonlinear dynamics in affective valence and arousal recognition," IEEE Trans. Affect. Comput., vol. 3, pp. 237-249, 2012.

[18] K. C. Chua, V. Chandran, U. R. Acharya, and C. M. Lim, "Cardiac state diagnosis using higher order spectra of heart rate variability," $J$. Med. Eng. Technol., vol. 32, pp. 145-155, 2008.

[19] S. M. Zhou, J. Q. Gan, and F. Sepulveda, "Classifying mental tasks based on features of higher-order statistics from EEG signals in braincomputer interface," Inf. Sci., vol. 178, pp. 1629-1640, 2008.

[20] C. L. Nikias and M. R. Raghuveer, "Bispectrum estimation: A digital signal processing framework," Proc. IEEE, vol. 75, pp. 869-891, 1987.

[21] C. J. Stam, W. de Haan, A. Daffertshofer, B. F. Jones, I. Manshanden, A. M. van Cappellen van Walsum, et al., "Graph theoretical analysis of magnetoencephalographic functional connectivity in Alzheimer's disease," Brain, vol. 132, pp. 213-224, 2009

[22] T. Koenig, L. Prichep, T. Dierks, D. Hubl, L. O. Wahlund, E. R. John, and V. Jelic, "Decreased EEG synchronization in Alzheimer's disease and mild cognitive impairment," Neurobiol. Aging, vol. 26, pp. 165$171,2005$.

[23] X. Delbeuck, M. van der Linden, and F. Collette, "Alzheimer's disease as a disconnection syndrome?," Neuropsychol. Rev., vol. 13, pp. 79-92, 2003.

[24] C. Lehmann, T. Koenig, V. Jelic, L. Prichep, R. E. John, L. Wahlund, et al., "Application and comparison of classification algorithms for recognition of Alzheimer's disease in electrical brain activity (EEG)," J. Neurosci. Methods, vol. 161, pp. 342-350, 2007. 